\title{
Sử dụng các ứng dụng họp hội nghị để dạy học trong mùa dịch Covid-19 tại Việt Nam
}

\section{Acceptance and use of video conferencing applications for teaching during Covid-19 pandemic in Vietnam}

\author{
Nguyễn Thanh Khương ${ }^{1 *}$ \\ ${ }^{1}$ Trường Đại học Luật Thành phố Hồ Chí Minh, Việt Nam \\ *Tác giả liên hệ, Email: ntkhuong@hcmulaw.edu.vn
}

\section{THÔNG TIN}

DOI:10.46223/HCMCOUJS. soci.vi....

Ngày nhận: 24/06/2021

Ngày nhận lại: 23/08/2021

Duyệt đăng: 24/08/2021

Tù khóa:

dạy trực tuyến; họp hội nghị; học trực tuyến; utaut2

Keywords:

online teaching; video conferece application; online learning; utaut2

\section{TÓM TẮT}

Úng dụng họp hội nghị là một công cụ được sử dụng phổ biến trong đào tạo từ xa. Sử dụng các ứng dụng họp hội nghị đã giải quyết được nhu cầu giảng dạy và học tập liên tục trong đại dịch Covid-19 tại Việt Nam. Với khung lý thuyết về chấp nhận và sử dụng công nghệ (UTAUT), nghiên cứu này nhằm xác định các yếu tố tác động đến việc sử dụng các ứng dụng họp hội nghị cho việc giảng dạy trong thời gian dịch Covid-19 diễn ra tại Việt Nam bởi các thầy, cô giáo. Bằng công cụ bảng hỏi trực tuyến trên Google Docs, nghiên cứu thu thập được 215 mẫu với 203 mẫu đạt yêu cầu được sử dụng, các biên quan sát và các mối quan hệ trong mô hình được phân tích qua từng bước gồm phân tích khám phá (EFA), phân tích khẳng định $(\mathrm{CFA})$ và phân tích bằng kỹ thuật mô hình cấu trúc tuyến tính (SEM). Các yếu tố bao gồm: kỳ vọng nổ lực, thói quen, động lực thụ hưởng tác động tích cực đến ý định sử dụng các ứng dụng họp trực tuyến và ý định sử dụng đồng thời giải thích được $65.5 \%$ về hành vi sử dụng các ứng họp hội nghị $\left(\mathrm{R}^{2}=0.655\right)$ để giảng dạy.

\section{ABSTRACT}

Applications for conferences are a common tool in distance learning. During the Covid-19 pandemic in Vietnam, teaching and learning was resolved by using conference applications. The purpose of this study is to identify the variables that influence the use of conference software in Vietnam by teachers during the Covid-19 epidemic. The study gathered 215 samples with 203 acceptable samples used, and the model's relationships and observations were explored through each step, including exploratory analysis, confirmatory analysis, and Structural Equation Modeling (SEM). The use of meeting applications, both in terms of having the intention to use and actually using them, is positively affected by the presence of factors like hedomic motivation, habits, and effort expectations with explaining $65.5 \%$ of usage behavior $\left(\mathrm{R}^{2}=0.655\right)$. 


\section{Giới thiệu}

Theo thống kê của United Nations năm 2020, có hơn 1.6 tỉ người học chịu tác động trực tiếp bởi dịch bệnh Covid-19 trên 190 quốc gia trên thế giới (Brief, 2020). Tại Việt Nam, trong quá trình dịch bệnh Covid-19 bùng phát, trường học ở tất cả các cấp học buộc phải đóng cửa theo chỉ thị 16/CT-TTg của Thủ tướng về việc thực hiện cách ly toàn xã hội (Prime Minister, 2020). Bộ Giáo dục và Đào tạo đã có các công văn chỉ đạo bao gồm công văn 1061/BGDĐT-GDTrH về việc "Hương dẫn dạy học qua Internet, trên truyền hình đối với cơ sở giáo duc (CSGD) phổ thông, CSGD thuờng xuyên trong thờ gian hoc sinh nghi hoc ở trương vì Covid-19 năm hoc 2019 - 2020 " (Pham \& Ho, 2020) ngày 25 tháng 03 năm 2020. Các giải pháp được đề cập đến bao gồm việc học tập từ xa thông qua các hệ thống như E-learning, học qua truyền hình, học thông qua các ứng dụng họp hội nghị, ... Việc thay đổi hình thức dạy và học khi giãn cách xã hội là một thách thức lớn đối với ngành giáo dục Việt Nam. Các ứng dụng họp hội nghị được sử dụng phổ biến phục vụ cho công tác đào tạo trực tuyến nhằm duy trì và đảm bảo chất lượng học tập của người học. Với việc kết hợp với các hệ thống quản trị nội dụng (LMS), các ứng dụng họp hội nghị đã trở thành công cụ hữu ích giúp cho người dạy trên môi trường trực tuyến. Đây là đối tượng chủ chốt quyết định đến chất lượng và hiệu quả của giờ học khi trực tiếp sử dụng các chức năng của phần mềm nhằm tối ưu hóa để đạt kết quả cao của giờ học. Nhận thấy được vai trò quan trọng trong việc hình thành ý định và hành vi sử dụng các ứng dụng họp hội nghị của người dạy giáo viên cho việc hướng dẫn nói riêng và phát triển giáo dục trực tuyến nói chung. Nghiên cứu này sử dụng khung lý thuyết thống nhất chấp nhận và sử dụng công nghệ (UTAUT) (Venkatesh, Morris, Davis, \& Davis, 2003; Venkatesh, Thong, \& Xu, 2012) nhằm khám phá ra những yếu tố tác động đến ý định và hành vi sử dụng của người dạy khi sử dụng các ứng dụng họp hội nghị để giảng dạy trong mùa dịch Covid19. Đối tượng và phạm vi nghiên cứu là người dạy (thầy, cô giáo) đã từng sử dụng các ứng dụng họp hội nghị để dạy học trong thời gian dịch Covid-19 bùng phát tại Việt Nam.

\section{Cơ sở lý thuyết}

\subsection{Họ hội nghị}

Họp hội nghị là cuộc họp trong đó những người tham gia ở những địa điểm khác nhau có thể giao tiếp với nhau bằng âm thanh và hình ảnh (Stevenson, 2010). Trong mùa dịch Covid-19 diễn ra, các công cụ họp hội nghị được sử dụng rộng rãi như là các ứng dụng họp hội nghị miễn phí như: Zoom, Google Meet, Ms Teams, Skype, ...

Hội nghị là một công cụ giao tiếp công nghệ cao cho phép bạn tăng hiệu quả kinh doanh, đơn giản hóa và đẩy nhanh quá trình ra quyết định, đồng thời cũng giảm chi phí đi lại cho khách hàng và nhân viên công ty trong quá trình này. Trong lĩnh vực giáo dục, các cuộc họp và hội nghị giúp cho sự phát triển không ngừng của các hoạt động dạy và học trong thời kỳ đại dịch Covid19, mang lại sự tương tác trực tiếp và là tiền đề cho sự phát triển của các chương trình giáo dục.

\subsection{Mô hình nghiên cúu}

Venkatesh và cộng sự (2003) phát triển mô hình lý thuyết thống nhất và chấp nhận sử dụng công nghệ (UTAUT) nhằm giải thích mục đích và hành vi cho việc sử dụng hệ thống thông tin (Venkatesh et al., 2003). Các lý thuyết bao gồm: lý thuyết hành động hợp lý (TRA) (Fishbein \& Ajzen, 1977; Fishbein, Jaccard, Davidson, Ajzen, \& Loken, 1980); lý thuyết hành vi dự định (TPB) (Ajzen, 1985); mô hình chấp nhận công nghệ (TAM) (Davis, 1989); mô hình chấp nhận công nghệ kêt hợp với lí thuyết hành vi dự định (C-TAM-TPB) (Taylor \& Todd, 1995), lí thuyết phổ biến sự đổi mới (IDT) (Moore \& Benbasat, 1991), mô hình động lực thúc đẩy (MM) (Davis, Bagozzi, \& Warshaw, 1992), mô hình sử dụng máy tính (MPCU) (Thompson, Higgins, \& Howell, 1991), và lí thuyết nhận thức xã hội (SCT) (Compeau \& Higgins, 1995) được sử dụng trong quá trình hình thành và phát triển ra UTAUT. Kỳ vọng hiệu quả, kỳ vọng nổ lực, ảnh hưởng xã hội và điều kiện 
thuận lợi là các yếu tố được xây dựng dựa trên các lý thuyết TRA, TPB và TAM liên quan đến ý định và hành vi sử dụng hệ thống thông tin trong mô hình UTAUT. Năm 2012, ông và cộng sự tiếp tục mở rộng thêm UTAUT thành UTAUT2 bằng cách tích hợp thêm các yếu tố, cụ thể như: động lực thụ hưởng, giá trị giá cả và thói quen (Venkatesh et al., 2012).

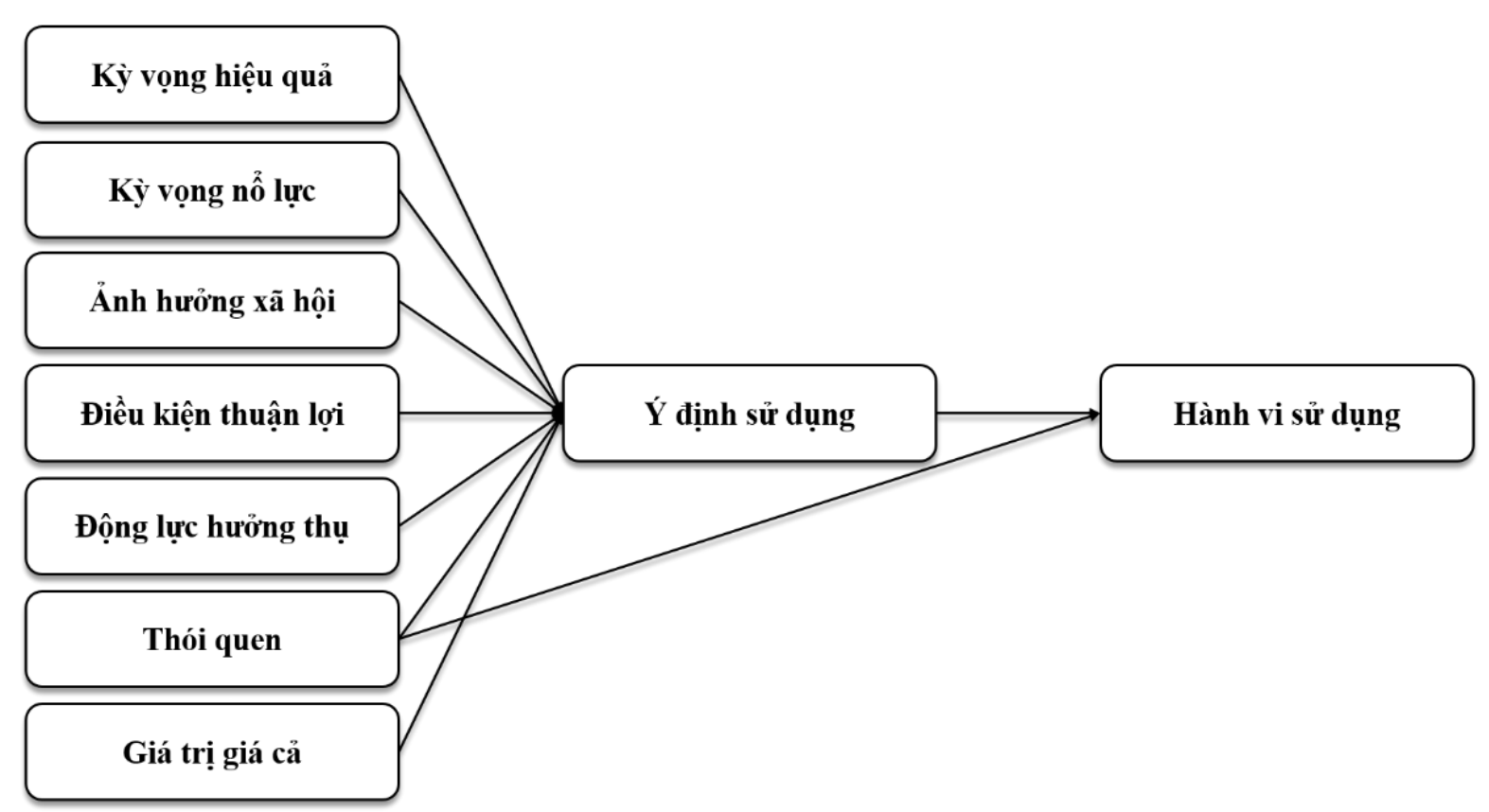

Hình 1. Mô hình lý thuyết thống nhất và chấp nhận sử dụng công nghệ (UTAUT)

\subsection{Một số nghiên cúu trước đây}

Một số nghiên cứu chỉ ra rằng việc sử dụng hệ thống họp hội nghị trong đào tạo từ xa và trong thời gian giãn cách do dịch Covid-19 bùng phát mang lại các kết quả cao và sự hài lòng của người học (xem Bảng 1). Sử dụng các hệ thống họp hội nghị hoặc phần mềm họp hội nghị đã góp phần đảm bảo việc học tập xuyên suốt.

\section{Bảng 1}

Các nghiên cứu trước đây về sử dụng họp hội nghị trong đào tạo

\section{Nghiên cứu}

(Townsend, Demarie, \& Hendrickson, 2001)

(MacLaughlin, Supemaw, \& Howard, 2004)

(Alkhaldi, Yusof, \& Aziz, 2012)

(Fatani, 2020)

\section{Nội dung}

Nghiên cứu về họp hội nghị trên máy tính để bàn trong nhóm làm việc ảo: dự đoán, đánh giá hệ thống và hiệu suất. Người học đạt đạt kết quả cao khi sử dụng hệ thống họp hội nghị.

Tác động của công nghệ họp hội nghị trong đào tạo từ xa đến kết quả học tập của sinh viên. Kết quả học tập trên họp hội nghị như học tập truyền thống.

Nghiên cứu về việc sử dụng họp hội nghị trong đào tạo người dùng trong các tổ chức tại Jordan. Cảm nhận dễ sử dụng hưởng hưởng trực tiếp đến người dùng khi dùng họp hội nghị.

Sự hài lòng của người học khi sử dụng họp hội nghị trong mùa dịch Covid-19. Sự hài lòng của người học phụ thuộc vào phương pháp giảng dạy, nhận thức và sự hiện diện xã hội chứ không phụ thuộc vào công nghệ. 
Việc học tập sử dụng họp hội nghị dưới góc nhìn và thái độ của

(Ngo, Nguyen, \& Tran, nữ sinh viên tại Việt Nam trong suốt dịch Covid-19. Cảm nhận 2020) dễ sử dụng và hữu ích ảnh hưởng tích cực trong việc sử dụng họp hội nghị của sinh viên nữ.

(Nguyen, Pho, Luong, \& Cao, 2021) Sử dụng họp hội nghị trong đào tạo từ xa tại Việt Nam trong đại dịch Covid-19. Cảm nhận hữu ích ảnh hưởng tích cực trong việc sử dụng họp hội nghị trong đào tạo từ xa tại Việt Nam.

Nguồn: Tác giả tổng hợp

\subsection{Các giả thuyết}

2.4.1. Kỳ vọng hiệu quả (HQ): được giải thích là việc đạt được lợi ích trong công việc khi người dùng tin tưởng vào việc sử dụng một hệ thống chuyên môn nào đó (Venkatesh et al., 2003). Do đó, giả thuyết H1 như sau:

\section{H1: Có mối liên hệ duơng giũa $H Q$ và $Y D$}

2.4.2. Kỳ vọng nỗ lực (NL): được định nghĩa là việc sử dụng hệ thống thông tin với mức độ dễ kết hợp (Venkatesh et al., 2003). Amoako-Gyampah và Salam (2004) nhận định người dùng sẽ có ý định sử dụng một hệ thống thông tin khi họ nổ lực (Amoako-Gyampah \& Salam, 2004). Giả thuyết $\mathrm{H} 2$ như sau:

H2: Có mối liên hệ duơng giũa $N L$ và $Y D$

2.4.3. Anh hương xã họi $(X H)$ : là việc mà người dùng bị ảnh hưởng bởi những người quan trọng xung quanh họ khi sử dụng một hệ thống thông tin (Venkatesh et al., 2003). Giả thuyết H3 như sau:

\section{H3: Có mối liên hệ duơng giũ̃a XH và YD}

2.4.4. Điều kiện thuận lợi $(D K)$ : là việc người dùng tin rằng mình được hỗ trợ và có cơ sở hạ tầng để sử dụng tốt hệ thống thông tin (Venkatesh et al., 2003). Do đó, ta có giả thuyết H4:

H4: Có mối liên hệ duơng giũua $D K$ và $Y D$

2.4.5. Động lục hương thu $(D L)$ : là sự thỏa mãn hay hạnh phúc có được từ việc sử dụng hệ thống (Brown \& Venkatesh, 2005). Giả thuyết H5 là:

H5: Có mối liên hệ dương giũua DL và YD

2.4.6. Thói quen (TQ): là hành vi của người dùng sẽ ảnh hưởng trực tiếp đến việc sử dụng hệ thống thông tin (Venkatesh et al., 2012). Do đó, ta có giả thuyết H6, H7:

H6: Có mối liên hệ duong giũa TQ và $Y D$

H7: Có mối liên hệ duơng giũa TQ và $S D$

2.4.7. Giá trị giá cả $(G C)$ : là lợi ích có được từ việc sử dụng hệ thống thông tin so với các chi phí phải bỏ ra (Venkatesh et al., 2012). Do đó, ta có giả thuyết H8:

H8: Có mối liên hệ duơng giữa GC và $Y D$

2.4.8. Ý định sử dụng $(Y D)$ : là ý định của một cá nhân cho hành động của một hành vi thực tế, cụ thể là hành vi sử dụng hệ thống (Mun, Jackson, Park, \& Probst, 2006). Do đó, ta có giả thuyết H9:

H9: Có mối liên hệ duơng giữa YD và SD

\section{Phương pháp nghiên cứu}


Phương pháp nghiên cứu định tính và phương pháp nghiên cứu định lượng được sử dụng trong nghiên cứu. Ở giai đoạn nghiên cứu định tính, thang đo nháp được kiểm tra sơ bộ để trở thành thang đo chính thức thông qua thảo luận tay đôi và thảo luận nhóm với giáo viên; người đã sử dụng các ứng dụng họp hội nghị để dạy học trong thời gian giãn cách. Trong giai đoạn nghiên cứu định lượng, nghiên cứu sử dụng bảng hỏi trực tuyến được gửi đến các diễn đàn giáo viên, nhóm học thuật của thầy, cô giáo và trực tiếp qua email với các biến quan sát được kiểm tra bằng thang đo Likert 5 điểm tương ứng mức thấp nhất là 1 - Hoàn toàn không đồng ý và mức cao nhất là 5 - Hoàn toàn đồng ý. Qua quá trình sàn lọc, loại bỏ các mẫu dữ liệu không phù hợp có 203 mẫu được sử dụng trong tổng số 215 mẫu thu thập được của 29 biến quan sát. Các phương pháp phân tích nhân tố khám phá (EFA), nhân tố khẳng định (CFA) và mô hình cấu trúc tuyến tính (SEM) được sử dụng với phần mềm SPSS và $\mathrm{AMOS}$ để kiểm định mô hình và các giả thuyết.

\section{Bảng 2}

Mô tả dữ liệu, phương sai trích trung bình và bình phương hệ số tương quan

\begin{tabular}{lcccccccccc}
\hline & Trung bình & Độ lệch chuẩn & HQ & NL & DK & SD & YD & TQ & XH & DL \\
\hline HQ & 4.26 & 0.68 & $\mathbf{0 . 7 3}$ & & & & & & & \\
NL & 3.90 & 0.87 & 0.34 & $\mathbf{0 . 8 2}$ & & & & & & \\
DK & 3.97 & 0.74 & 0.17 & 0.32 & $\mathbf{0 . 7 6}$ & & & & & \\
SD & 4.03 & 0.79 & 0.14 & 0.39 & 0.45 & $\mathbf{0 . 8 2}$ & & & & \\
YD & 4.22 & 0.75 & 0.26 & 0.40 & 0.37 & 0.62 & $\mathbf{0 . 8 4}$ & & & \\
TQ & 3.91 & 0.83 & 0.27 & 0.32 & 0.38 & 0.42 & 0.53 & $\mathbf{0 . 8 1}$ & & \\
XH & 3.97 & 0.87 & 0.25 & 0.25 & 0.33 & 0.22 & 0.34 & 0.40 & $\mathbf{0 . 7 9}$ & \\
DL & 3.96 & 0.77 & 0.22 & 0.43 & 0.49 & 0.46 & 0.51 & 0.56 & 0.35 & $\mathbf{0 . 7 4}$ \\
\hline
\end{tabular}

Nguồn: Kết quả từ nghiên cứu

Bảng 3

Thang đo và tổng hợp kết quả phân tích

\begin{tabular}{|c|c|c|c|c|c|c|}
\hline & \multirow{2}{*}{ Biến quan sát } & & \multicolumn{4}{|c|}{ Hệ số tải } \\
\hline & & & EFA & CFA & $\mathbf{C R}$ & AVE \\
\hline \multicolumn{7}{|c|}{ Kỳ vọng hiệu quả: HQ } \\
\hline 1 & $\begin{array}{l}\text { Sẵn sàng sử dụng các ứng dụng hệ thống } \\
\text { họp hội nghị để giảng dạy trực tuyến. }\end{array}$ & HQ1 & 0.695 & 0.745 & & \\
\hline 2 & $\begin{array}{l}\text { Sử dụng các ứng dụng họp hội nghị rất thú } \\
\text { vị. }\end{array}$ & HQ2 & 0.641 & 0.604 & 0.77 & 0.53 \\
\hline 3 & $\begin{array}{l}\text { Các ứng dụng họp hội nghị tích hợp nhiều } \\
\text { chức năng hỗ trợ giảng dạy trực tuyến. }\end{array}$ & HQ3 & 0.842 & 0.820 & & \\
\hline
\end{tabular}

Kỳ vọng nỗ lực: NL

4 Sử dụng các ứng dụng họp hội nghị để

giảng dạy thật dễ dàng.

$\begin{array}{lllll}\text { NL1 } & 0.899 & 0.868 & 0.89 & 0.68\end{array}$ 


\begin{tabular}{|c|c|c|c|c|c|c|}
\hline & \multirow{2}{*}{\multicolumn{2}{|c|}{ Biến quan sát }} & \multicolumn{4}{|c|}{ Hệ số tải } \\
\hline & & & EFA & CFA & $\mathbf{C R}$ & AVE \\
\hline 5 & $\begin{array}{l}\text { Tôi hiểu rõ cách thức làm việc trên các ứng } \\
\text { dụng họp hội nghị. }\end{array}$ & NL2 & 0.787 & 0.831 & & \\
\hline 6 & $\begin{array}{l}\text { Các ứng dụng họp hội nghị cung cấp hướng } \\
\text { dẫn đầy đủ. }\end{array}$ & NL3 & 0.710 & 0.752 & & \\
\hline 7 & $\begin{array}{l}\text { Học cách sử dụng hệ thống họp hội nghị } \\
\text { thật dễ dàng. }\end{array}$ & NL4 & 0.872 & 0.840 & & \\
\hline \multicolumn{7}{|c|}{ Ảnh hưởng xã hội: XH } \\
\hline 8 & $\begin{array}{l}\text { Các đồng nghiệp xung quanh tôi nghĩ việc } \\
\text { sử dụng hệ thống hộp hội nghị cho việc } \\
\text { giảng dạy trực tuyến sẽ đạt hiệu quả cao } \\
\text { hơn trong mùa Covid-19 }\end{array}$ & XH1 & 0.753 & 0.755 & & \\
\hline 9 & $\begin{array}{l}\text { Các trường học khác cũng sử dụng hệ } \\
\text { thống họp hội nghị cho công việc giảng dạy } \\
\text { trực tuyến của họ trong mùa Covid-19 }\end{array}$ & XH2 & 0.895 & 0.883 & 0.834 & 0.627 \\
\hline 10 & $\begin{array}{l}\text { Quản lý nghĩ rằng sử dụng họp hội nghị để } \\
\text { giảng dạy trực tuyến để tăng tính tương tác } \\
\text { trong mùa Covid-19 }\end{array}$ & XH3 & 0.729 & 0.729 & & \\
\hline
\end{tabular}

\section{Động lực hưởng thụ: DL}

Tôi có sẵn mọi thông tin cho việc giảng dạy

11 trực tuyến khi sử dụng hệ thống họp hội

DL1 $0.647 \quad 0.711$ nghị.

Sử dụng hệ thống họp hội nghị giúp tôi

12 tăng khả năng tương tác với học trò trên môi trường trực tuyến.

$\begin{array}{lllll}\text { DL2 } & 0.705 & 0.704 & 0.78 & 0.54\end{array}$

Dễ dàng có được sự giúp đỡ của ngưới

13 khác khi sử dụng hệ thống họp hội nghị để giảng dạy.

\section{Thói quen: TQ}

Sử dụng hệ thống họp hội nghị cho công

14 việc giảng dạy trực tuyến là thói quen của tôi trong mùa Covid-19.

TQ1 $0.770 \quad 0.804$

5 Dạy học với các ứng dụng họp hội nghị rất thoải mái.

TQ2

0.781

0.823

$0.85 \quad 0.65$

16 Sử dụng liên tục các ứng dụng họp hội nghị trong mùa dịch Covid-19.

TQ3 $\quad 0.771$

0.791

\section{Điều kiện thuận lọii: DK}

17 Sử dụng hệ thống họp hội nghị để giảng dạy trực tuyến trong điều kiện thuận lợi.

DK1 $0.686 \quad 0.747$

Có điều kiện thuận lợi về tìm kiếm video hướng dẫn để học tập và sử dụng hệ thống họp hội nghị cho việc giảng dạy trực tuyến

DK2 $\quad 0.810 \quad 0.787$ 


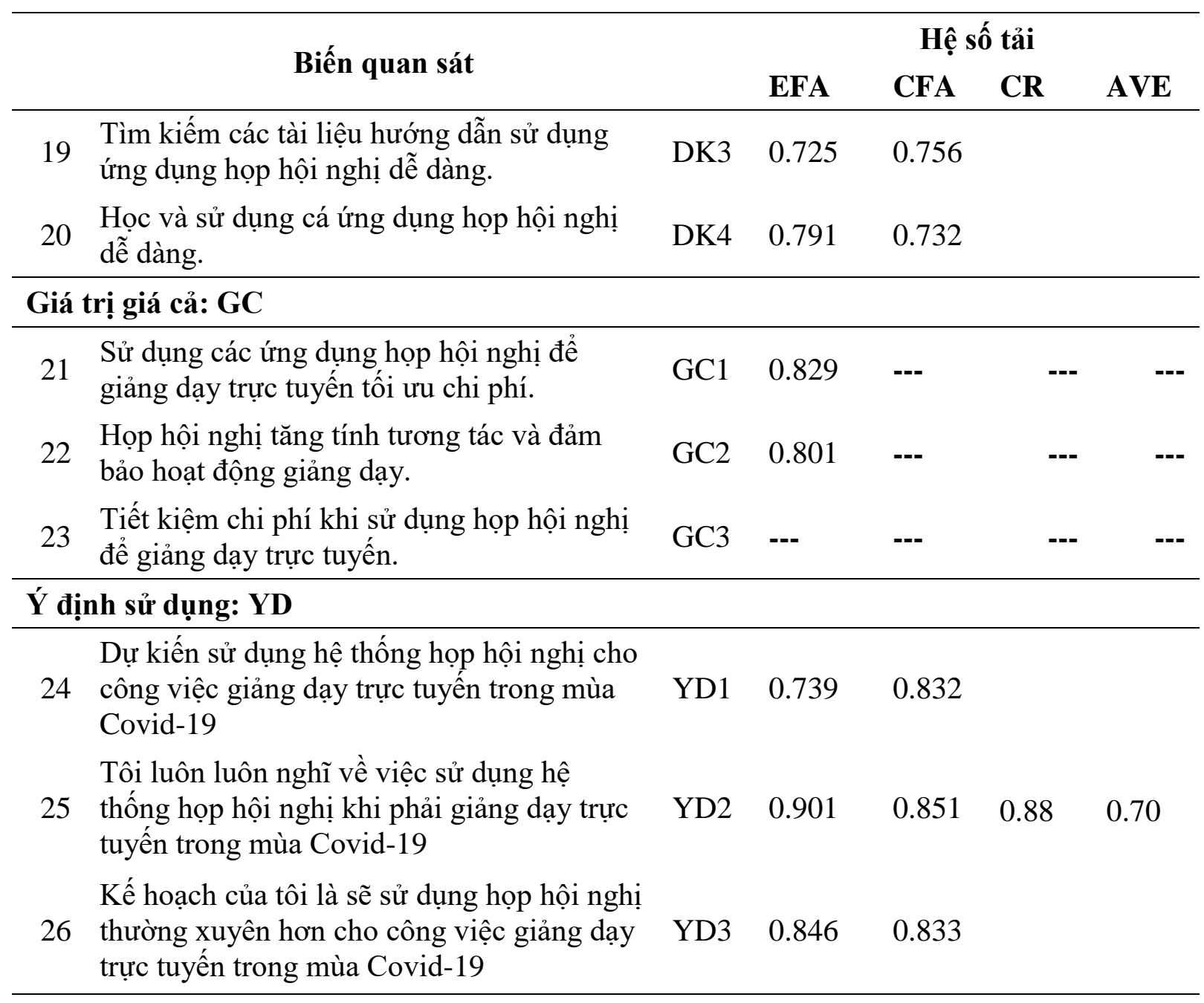

\section{Hành vi sử dụng: HV}

Tôi luôn sử dụng hệ thống họp hội nghị cho

27 công việc giảng dạy trực tuyến trong mùa HV1 $0.748 \quad 0.765$

Covid-19

Tôi làm tương tác tốt hơn với học sinh

28 thông qua hệ thống họp hội nghị trong mùa $\begin{array}{lllll}\text { HV2 } & 0.799 & 0.85 & 0.86 & 0.67\end{array}$

Covid-19

Sử dụng hệ thống họp hội nghị để giảng

29 dạy trực tuyến trong màu Covid-19 cho tôi $\quad$ HV3 $0.937 \quad 0.834$

nhiều trải nghiệm thú vị

Nguồn: Kết quả từ nghiên cứu

\section{Kết quả nghiên cứu và thảo luận}

\subsection{Thống kê mô tả}

- Phần mềm họp hội nghị: Google Meet: 11.3\%, MS Team: 31.5\%, Zoom: 53.7\% và phần mềm khác: $3.4 \%$;

- Bậc giảng dạy: Tiểu học: 9.4\%, THCS: 22.7\%, THPT: 51.2\% và Đại học: $16.7 \%$;

- Giới tính: Nam: 30.5\% và Nữ: 69.5\%; tuổi: $4.9 \%$;

- Độ tuổi: Từ 22 - 30 tuổi: $15.3 \%$, từ 31 - 40 tuổi: $50.2 \%$, từ 41 - 50 tuổi: $29.6 \%$ và trên 50

- Trình độ: Cao đẳng: 3.4\%, Đại học: 54.2\% và Sau đại học: 42.4\%; 
- Kinh nghiệm sử dụng CNTT: Từ 01 - 05 năm: 14.3\%, từ 06 - 10 năm: 29.1\%, từ 11 - 15 năm: $26.6 \%$, trên 15 năm: $30 \%$.

\subsection{Phân tích nhân tố khám phá (EFA)}

Ở lần đầu tiên, EFA bỏ đi biến GC3 của thành phần Giá trị giá cả do có hệ số tải nhân tố nhỏ hơn 0.5. Ở lần tiếp theo, EFA rút trích được trích được 09 nhân tố từ 28 biến như Bảng 2 được phân thành thành từng nhóm nhân tố trong ma trận xoay yếu tố. Hệ số tin cậy của các yếu tố thang đo phù hợp lớn hơn 0.7 (từ 0.757 đến 0.892) (Hair, Black, Babin, Anderson, \& Tatham, 2006).

\subsection{Phân tích nhân tố khẳng định (CFA)}

CFA lần 1 bỏ đi biến GC1 của thành phần Giá trị giá cả do hệ số tải nhân tố nhỏ hơn 0.5 . Do đó, thành phần của Giá trị giá cả $(\mathrm{GC})$ chỉ còn lại 01 biến để đo lường cho thành phần này nên thành phần GC bị loại ra khỏi CFA. Kiểm định mô hình đo lường với các chỉ số Chi-square $(\chi) / \mathrm{dF}$ $=1.197 ; \mathrm{GFI}=0.895 ; \mathrm{TLI}=0.975 ; \mathrm{CFI}=0.979$ và $\mathrm{RMSEA}=0.031$ nên thang đo tương thích với tập dữ liệu (Byrne, 2013). Hệ số tải CFA các biến từ 0.604 đến 0.883 . Các thang đo đạt được giá trị hội tụ khi có phương sai trích trung bình (AVE) lớn hơn 0.5 (từ 0.531 đến 0.703 ). Bình phương hệ số tương quan $\left(\mathrm{r}^{2}\right)$ nhỏ hơn AVE tương ứng nên các thang đo đạt giá trị phân biệt (Bảng 3 ).

\section{Bảng 4}

Mối quan hệ giũa các thành phần và kết quả kiểm định các giả thuyết

\begin{tabular}{ccccccccc}
\hline $\begin{array}{c}\text { Giả } \\
\text { thuyết }\end{array}$ & & & & Estimate & SE & CR & Mức ý nghĩa & Kết quả \\
\hline H1 & YD & $\leftarrow$ & HQ & 0.052 & 0.089 & 0.592 & 0.554 & Loại bỏ \\
H2 & YD & $\leftarrow$ & NL & $\mathbf{0 . 1 4 3}$ & 0.066 & 2.186 & $\mathbf{0 . 0 2 9}$ & Xác nhận \\
H3 & YD & $\leftarrow$ & XH & 0.056 & 0.067 & 0.835 & 0.404 & Loại bỏ \\
H4 & YD & $\leftarrow$ & DK & 0.097 & 0.089 & 1.1 & 0.271 & Loại bỏ \\
H5 & YD & $\leftarrow$ & DL & $\mathbf{0 . 2 0 7}$ & 0.107 & 1.932 & $\mathbf{0 . 0 5 3}$ & Xác nhận \\
H6 & YD & $\leftarrow$ & TQ & $\mathbf{0 . 2 7 4}$ & 0.09 & 3.043 & $\mathbf{0 . 0 0 2}$ & Xác nhận \\
H8 & SD & $\leftarrow$ & TQ & 0.135 & 0.083 & 1.617 & 0.106 & Loại bỏ \\
H9 & SD & $\leftarrow$ & YD & $\mathbf{0 . 5 7}$ & 0.093 & 6.144 & $* * *$ & Xác nhận \\
\hline
\end{tabular}

Nguồn: Kết quả từ nghiên cứu

\subsection{Phân tích mô hình cấu trúc tuyến tính (SEM)}

Với phương pháp ước lượng khả dĩ (ML) kết quả phân tích SEM như ở Bảng 3 bao gồm $\mathrm{TQ}, \mathrm{DL}$ và NL ảnh hưởng trực tiếp với các hệ số $\gamma$ lần lượt là $0.274(\mathrm{p}=0.002) ; 0.207(\mathrm{p}=0.053)$ và $0.143(\mathrm{p}=0.029)$ đến $\mathrm{YD}$. Vì vậy, các giả thuyết $\mathrm{H} 2, \mathrm{H} 5$ và H6 được xác nhận. Hơn nữa, yếu tố YD ảnh hưởng tích cực với hệ số $\gamma$ là $0.57(\mathrm{p}=0.000)$ đến $\mathrm{SD}$ nên giả thuyết $\mathrm{H} 9$ cũng được xác nhận. Sau khi lượt bỏ các giả thuyết không hỗ trợ, SEM mới với các chỉ số Chi-square $(\chi) / \mathrm{dF}=$ 1.326; GFI $=0.924 ;$ TLI $=0.977 ; \mathrm{CFI}=0.982$ và $\mathrm{RMSEA}=0.040$ nên thang đo tương thích với tập dữ liệu (Byrne, 2013) được trình bày như Hình 2. Các yếu tố TQ, DL và NL tác động tích cực 
- hệ số $\gamma$ lần lượt là $0.319(\mathrm{p}=0.000) ; 0.166(\mathrm{p}=0.008)$ và $0.262(\mathrm{p}=0.011)$ đến YD và YD có ảnh hưởng trực tiếp đến $\mathrm{SD}$ với hệ số $\gamma$ là $0.655(\mathrm{p}=0.000)$.

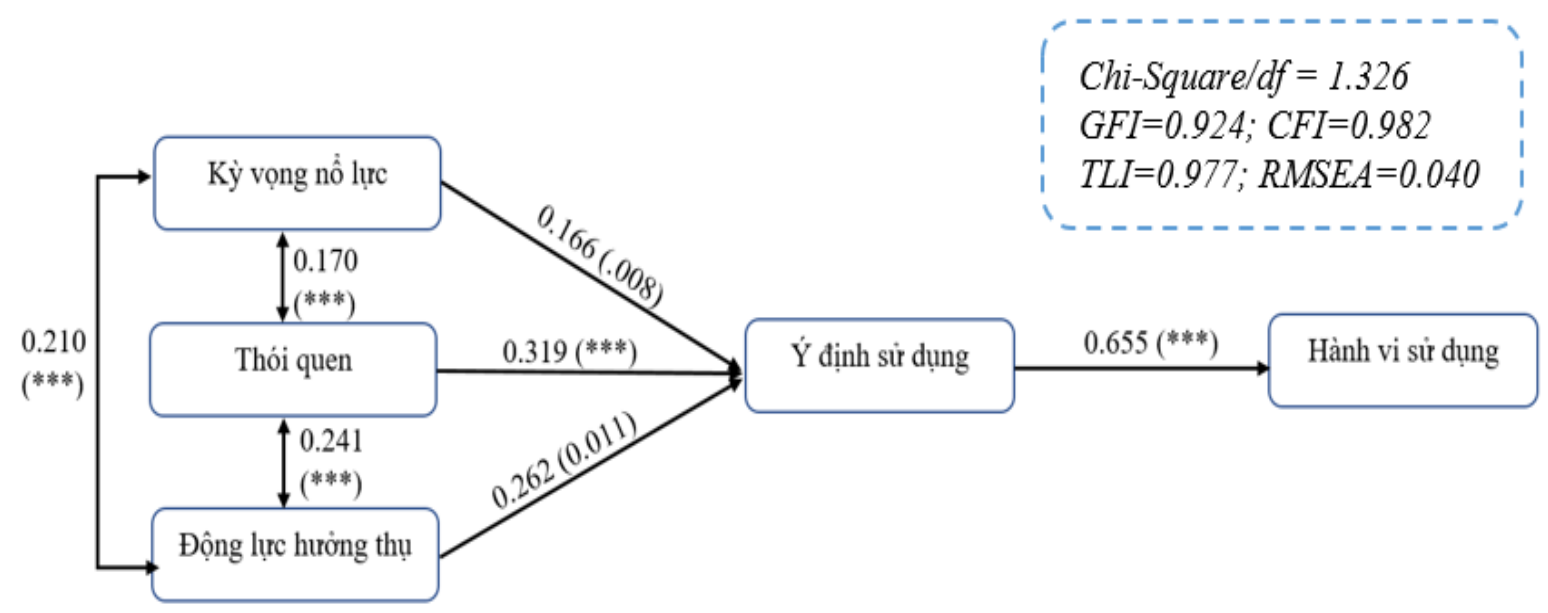

Hình 2. Kết quả kiểm định mô hình chấp nhận và sử dụng các ứng dụng họp hội nghị

\subsection{Thảo luận}

Nghiên cứu thông qua các bước phân tích EFA, CFA và SEM xác định có 04 giả thuyết phù hợp trong 09 giả thuyết được đưa ra. Các yếu tố độc lập và trung gian giải thích được khoảng $65.5 \%\left(\mathrm{R}^{2}=0.655\right)$ hành vi sử dụng các ứng dụng họp hội nghị của giáo viên phù hợp với các nghiên cứu trong mô hình UTAUT gốc (Venkatesh et al., 2012). Các yếu tố bao về EE, HM và TQ tác động tích cự đến $\mathrm{YD}$ và từ $\mathrm{YD}$ ảnh hưởng trực tiếp đến $\mathrm{SD}$ của hành vi người dạy trong quá trình sử dụng các ứng dụng họp hội nghị để giảng dạy trong thời gian giãn cách do dịch Covid19 tại Việt Nam.

\section{Kết luận và kiến nghị}

Nghiên cứu chỉ ra rằng các thang đo các biến độc lập, các biến phụ thuộc và biến sử dụng các ứng dụng họp hội nghị đều đảm bảo độ tin cậy. Thang đo đạt giá trị phân biệt và hội tụ qua phân tích EFA và CFA. Các yếu tố NL, DL, TQ và YD có quan hệ cấu trúc tuyến tính với SD giải thích được $65.5 \%$ hành vi của người dạy khi sử dụng các ứng dụng họp hội nghị để giảng dạy khi Covid-19 bùng phát.

Đối với hoạt động đào tạo trực tuyến, nghiên cứu giải thích được các yếu tố tác động đến việc sử dụng các ứng dụng họp hội nghị trong giảng dạy để việc học được diễn ra thông suốt trong thời gian dịch Covid-19 kéo dài. Sử dụng công cụ họp hội nghị là tất yếu trong đào tạo từ xa, để khai thác tốt ứng dụng này, giáo viên cần được hỗ trợ tập huấn và hướng dẫn để khai thác tốt các ứng dụng này trong việc vận hành nó. Bên cạnh đó, người dạy là nhân tố chủ chốt quyết định chất lượng của buổi học và sự hài lòng của người học trong quá trình sử dụng các ứng dụng họp hội nghị.

Đối với các nhà quản lý, các nhà hoạch định chính sách giáo dục, nghiên cứu còn là tài liệu tham khảo phù hợp cho việc đánh giá tác động sử dụng công nghệ họp hội nghị trong công tác đào tạo trực tuyến. Phát triển đào tạo trực tuyến không thể không gắn liền với việc dùng các công cụ họp hội nghị để trao đổi và giảng dạy giữa người dạy và người học. Vì thế, nhà quản lý cần có kế hoạch và chiến lược cụ thể trong việc khai thác và tích hợp các ứng dụng họp hội nghị với các hệ thống quản lý nội dung học tập phù hợp với từng cấp học nhằm đảm bảo và nâng cao chất lượng giảng dạy. 


\section{LÒ̀I CÁM ƠN}

Gửi lời cám ơn đến quý thầy. cô đã tham gia hỗ trợ trong quá trình hoàn thành nghiên cứu. Xin cám ơn trường Đại học Luật Thành phố Hồ Chí Minh đã tạo điều kiện để tôi hoàn thành công trình nghiên cứu của mình.

\section{Tài liệu tham khảo}

Ajzen, I. (1985). From intentions to actions: A theory of planned behavior. In Action control (pp. 11-39). New York, NY: Springer.

Alkhaldi, A. N., Yusof, Z. M., \& Aziz, M. (2012). Impact of user training and support on videoconferencing usage in organizations in Jordan using structural equation modelling analysis approach. World Applied Sciences Journal, 19(11), 1553-1562.

Amoako-Gyampah, K., \& Salam, A. F. (2004). An extension of the technology acceptance model in an ERP implementation environment. Information \& Management, 41(6), 731-745.

Brief, P. (2020). Education during Covid-19 and beyond. Retrieved October 20, 2020, from United Nations website: https://www.un.org/development/desa/dspd/2020/04/social-impact-ofCovid-19

Brown, S. A., \& Venkatesh, V. (2005). Model of adoption of technology in households: A baseline model test and extension incorporating household life cycle. MIS Quarterly, 29(3), 399-426.

Byrne, B. M. (2013). Structural equation modeling with LISREL, PRELIS, and SIMPLIS: Basic concepts, applications, and programming. Hove, East Sussex: Psychology Press.

Compeau, D. R., \& Higgins, C. A. (1995). Application of social cognitive theory to training for computer skills. Information Systems Research, 6(2), 118-143.

Davis, F. D. (1989). Perceived usefulness, perceived ease of use, and user acceptance of information technology. MIS Quarterly, 13(3), 319-340.

Davis, F. D., Bagozzi, R. P., \& Warshaw, P. R. (1992). Extrinsic and intrinsic motivation to use computers in the workplace 1. Journal of Applied Social Psychology, 22(14), 1111-1132.

Fatani, T. H. (2020). Student satisfaction with videoconferencing teaching quality during the Covid-19 pandemic. BMC Medical Education, 20(1), 1-8.

Fishbein, M., \& Ajzen, I. (1977). Belief, attitude, intention, and behavior: An introduction to theory and research. Philosophy and Rhetoric, 10(2), 130-132.

Fishbein, M., Jaccard, J., Davidson, A. R., Ajzen, I., \& Loken, B. (1980). Predicting and understanding family planning behaviors. Englewood Cliffs, NJ: Prentice Hall.

Hair, J. F., Black, W. C., Babin, B. J., Anderson, R. E., \& Tatham, R. L. (2006). Multivariate data analysis (6th ed.). Upper Saddle River, NJ: Prentice Hall.

MacLaughlin, E. J., Supemaw, R. B., \& Howard, K. A. (2004). Impact of distance learning using videoconferencing technology on student performance. American Journal of Pharmaceutical Education, 68(3), 1-6.

Moore, G. C., \& Benbasat, I. (1991). Development of an instrument to measure the perceptions of adopting an information technology innovation. Information Systems Research, 2(3), 192222.

Mun, Y. Y., Jackson, J. D., Park, J. S., \& Probst, J. C. (2006). Understanding information 
technology acceptance by individual professionals: Toward an integrative view. Information \& Management, 43(3), 350-363.

Ngo, T. T., Nguyen, T. T. T., \& Tran, G. T. (2020). Influence of learning by using video conferencing tools on perceptions and attitude of Vietnamese female students in Covid-19 $\begin{array}{llll}\text { pandemic. } & \text { Retrieved } \quad \text { August } & \text { 2020, from }\end{array}$ https://papers.ssrn.com/sol3/papers.cfm?abstract_id=3697029

Nguyen, A. X., Pho, H. D., Luong, H. D., \& Cao, A. X. T. (2021). Vietnamese students'acceptance of using video conferencing tools in distance learning in Covid-19 pandemic. Turkish Online Journal of Distance Education, 22(3), 139-162.

Pham, H. H., \& Ho, T. T. H. (2020). Toward a 'new normal'with e-learning in Vietnamese higher education during the post Covid-19 pandemic. Higher Education Research \& Development, 39(7), 1327-1331.

Prime Minister. (2020). Chỉ thị 06/CT-TTg ngày 31 tháng 03 năm 2020 về thục hiện các biện pháp cấp bách phòng, chống dịch Covid-19 [Directive 06/CT-TTg dated March 31, 2020 on the implementation of urgent measures to prevent and control the Covid-19 epidemic]. Retrieved February 10, 2020, from http://vanban.chinhphu.vn/portal/page/portal/chinhphu/hethongvanban?class_id=2\&_page =1\&mode=detail\&document_id=199607

Stevenson, A. (2010). Oxford dictionary of English. New York, NY: Oxford University Press.

Taylor, S., \& Todd, P. A. (1995). Understanding information technology usage: A test of competing models. Information Systems Research, 6(2), 144-176.

Thompson, R. L., Higgins, C. A., \& Howell, J. M. (1991). Personal computing: Toward a conceptual model of utilization. MIS Quarterly, 15(1), 125-143.

Townsend, A. M., Demarie, S. M., \& Hendrickson, A. R. (2001). Desktop video conferencing in virtual workgroups: Anticipation, system evaluation and performance. Information Systems Journal, 11(3), 213-227.

Venkatesh, V., Morris, M. G., Davis, G. B., \& Davis, F. D. (2003). User acceptance of information technology: Toward a unified view. MIS Quarterly, 27(3), 425-478.

Venkatesh, V., Thong, J. Y., \& Xu, X. (2012). Consumer acceptance and use of information technology: extending the unified theory of acceptance and use of technology. MIS Quarterly, 36(1), 157-178.

Creative Commons Attribution-NonCommercial 4.0 International License. 